1997-01-01

\title{
Extracting the Grain Boundary Character/Free Energy Relationship from the Microstructure: Pure $<100>$ and $<111>$ Tilt Boundaries
}

\author{
Brent L. Adams \\ b_I_adams@byu.edu \\ S. Costiner \\ D. Kinderlehrer \\ W. W. Mullins \\ Shlomo Ta'asan
}

Follow this and additional works at: https://scholarsarchive.byu.edu/facpub

Part of the Mechanical Engineering Commons

Original Publication Citation

Materials Research Society Symposium Proc. Vol. 472 p. 15-111, 1997

BYU ScholarsArchive Citation

Adams, Brent L.; Costiner, S.; Kinderlehrer, D.; Mullins, W. W.; and Ta'asan, Shlomo, "Extracting the Grain Boundary Character/Free Energy Relationship from the Microstructure: Pure $<100>$ and $<111>$ Tilt Boundaries" (1997). Faculty Publications. 1137.

https://scholarsarchive.byu.edu/facpub/1137

This Peer-Reviewed Article is brought to you for free and open access by BYU ScholarsArchive. It has been accepted for inclusion in Faculty Publications by an authorized administrator of BYU ScholarsArchive. For more information, please contact ellen_amatangelo@byu.edu. 


\title{
EXTRACTING THE GRAIN BOUNDARY CHARACTER/FREE ENERGY RELATIONSHIP FROM THE MICROSTRUCTURE: PURE <100> AND <111> TILT BOUNDARIES
}

\author{
B. ADAMS*, D. KINDERLEHRER**, S. TA'ASAN**, W.W. MULLINS*, S. COSTINER** \\ *Materials Science and Engineering, Carnegie Mellon University, Pittsburgh, PA 15213-3890 \\ **Mathematical Sciences, Carnegie Mellon University, Pittsburgh, PA 15213-3890
}

\section{ABSTRACT}

Analysis is described to extract the excess free energy of grain boundaries from the mesotexture of well-equilibrated polycrystalline samples. The approach is based upon the force and torque balances at triple junctions described in the classical work of Herring [1]. The main advantage of the approach is that the free energy function is obtained over the full fundamental zone of grain boundary types. For the purposes of exposition, the method is described for specified two-parameter hypersurfaces in the fundamental zone for cubic polycrystals (the sets of pure $<100>$ and $<111>$ tilt boundaries) and the free energy is assumed to depend only upon the lattice misorientation. Results from testbed simulations are presented.

\section{INTRODUCTION}

Orientation Imaging Microscopy (OIM) [2] reveals a wealth of information about polycrystalline microstructure at the mesoscale. Limited by typical spatial and angular resolutions of $\sim 100 \mathrm{~nm}$ and $0.5^{\circ}$, the technique is incapable of resolving individual dislocations. However, OIM is ideal for rapidly probing the crystallographic character of interfaces and grain boundaries. Since OIM scans of the mesotexture over areas of $1 \mathrm{~cm}^{2}$ are feasible, the data obtained is distinguished by its extent and reliability.

This paper describes an emerging area of work aimed at recovering information about the properties and behavior of grain boundaries from geometrical information carried in the mesostructure. The local physico-chemical characteristics of grain boundaries are known to depend strongly upon lattice misorientation and the orientation of the interface plane. That the most important properties of grain boundaries cannot be measured directly has been a serious impediment to progress in understanding structure-sensitive properties. 
In this paper the focus is on the excess free energy. The geometrical and crystallographic features of an isolated grain boundary are referred to its "character" or "type." What is sought is an association between grain boundary type and its free energy for the entire fundamental zone of distinctive types that naturally occur in polycrystals. Such associations influence many important macroscopic properties through the details of their distribution and connectivity in the semicontinuous grain boundary network (c.f. Lejcek and Hofmann [3]). They also establish the subsequent evolution of microstructure through a complex interplay of the thermodynamic driving forces and kinetic constraints which determine the motions of grain boundaries.

The equilibrium thermodynamics of triple junctions is reviewed, and Herring's relations are recalled. Application to selected two-parameter hypersurfaces of the fundamental zone of single phase cubic grain boundaries is described. A statistical, multiscale method is introduced for extracting the free energy from mesostructural data. The results from testbed simulations comprising $1.3 \times 10^{5}$ triple junctions are presented.

\section{EQUILIBRIUM THERMODYNAMICS OF TRIPLE JUNCTIONS}

Gibbs [4] introduced the relevant thermodynamics a century ago. The important entity is the interface excess free energy (per unit area) $\sigma$, which (in the absence of interfacial strain) is a function of temperature $T$, pressure $P$, the chemical potentials of each component $\mu_{i}$, misorientation of the lattice between the adjacent grains $\Delta g$, and the unit normal describing inclination of the boundary $\hat{n}$. The main task is to determine the functional dependence of $\sigma$ on the crystallographic parameters $\Delta g, \hat{n}$ for selected $T, P$ and $\mu_{i}$.

Triple junctions are presumed to be equilibrated under time and temperature combinations such that diffusion distances lie within the mesoscale range that is now accessible by tomographic OIM $(>100 \mathrm{~nm})$. Under such conditions the measurable geometry of the junction reflects a balance of the forces and torques acting on the adjacent boundaries. These are intimately related to the excess free energies associated with the boundaries.

Following Herring [1,5] each equilibrium triple junction is described by a system of two independent equations in six unknowns (three unknown interface energies, and three unknown torques):

$$
\sum_{j=1}^{3}\left\{\sigma_{j} \hat{b}_{j}+\left(\partial \sigma_{j} / \partial \chi\right) \hat{n}_{j}\right\}=\overrightarrow{0}
$$

Here $\hat{n}_{j}$ is the unit boundary normal associated with the jth boundary in the triple junction. Similarly $\hat{b}_{j}$ is a unit vector lying perpendicular in the jth boundary and perpendicular to the triple 
line $\hat{l}=\hat{n}_{j} \otimes \hat{b}_{j}$ which is common to all three adjacent boundaries. The geometry is illustrated in Figure 1. The angle $\chi$ in relation (1) is defined to be the right-handed angle of rotation about $\hat{l}$ associated with the jth boundary. (This changes two of the dihedral angles in the set $\chi_{12}, \chi_{13}, \chi_{23}$, which are defined in Figure 1.)

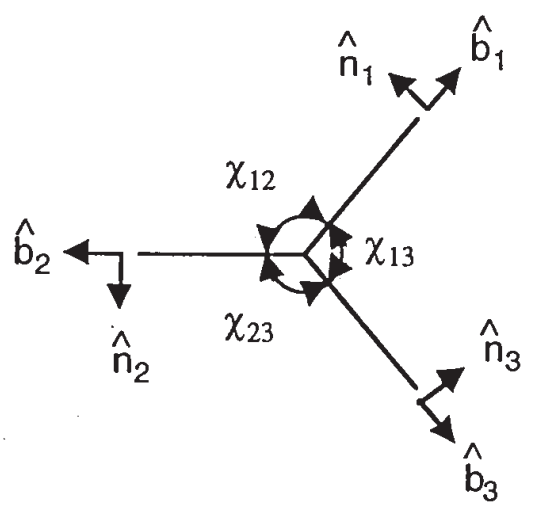

Fig-1 Parameters defining the geometric character of a triple junction.

\section{SELECTED TWO-DIMENSIONAL HYPERSURFACES OF THE FUNDAMENTAL ZONE}

Here the focus is upon attention on selected two-dimensional hypersurfaces of the fundamental zone. The cases of interest comprise the sets of $\langle 100\rangle$ and $\langle 111\rangle$ pure tilt boundaries in cubic polycrystals. These hypersurfaces approximate conditions of considerable technological importance, including $<100\rangle$ fiber-textured columnar microstructures obtained by directional solidification, and $\langle 111\rangle$ fiber-textured thin films where the energy tends towards a minimum by alignment of the boundaries nearly perpendicular to the underlying substrate. Both cases constitute microstructures whose characteristics lie within a small distance of the idealized hypersurfaces.

The Hypersurface for $<100>$ Tilt Boundaries : Let elements of the set of crystallographic planes containing a common [001] axis be identified by the angle $\phi$, which fixes the inclination of the plane (in the right-handed sense about [001]) relative to the [100] direction. Thus, the inclination parameter $\hat{n}$ in the general formulation is replaced by the angle $\phi$. For the case of interest here this set of boundaries possesses the point symmetry subgroup $D_{4 h}$. Considering the symmetry reduces the range of physically distinctive inclination angles to $0 \leq \phi \leq \pi / 4$. 
Elements of the hypersurface for $\langle 100\rangle$ tilt boundaries consist of a pair of crystallographic planes, sharing a common [001] axis, defined by crystallographic inclinations $\phi_{1}$ and $\phi_{2}$, and joined together such that their crystallographic misorientation is a pure rotation about [001] of angle $\Delta \phi=\mid \phi_{1}-\phi_{2}$ l. Thus, $\Delta \mathrm{g}$ reduces to $\Delta \phi$. Since, from an energetic point of view it does not matter which of the two crystallographic planes has crystallographic inclination $\phi_{1}$ and which has inclination $\phi_{2}$, the hypersurface $\mathcal{K}_{<100>}$ defining all pure $<100>$ tilt boundaries is given by the set:

$$
\mathcal{H}_{<100>}=\left\{\left(\phi_{1}, \phi_{2}\right): 0 \leq \phi_{1} \leq \pi / 4,0 \leq \phi_{2} \leq \phi_{1}\right\}
$$

Note that for pairs with $\phi_{2}=\phi_{1}$ the free energy must vanish.

The Hypersurface for $<111>$ Tilt Boundaries : Following the same notation, let elements of the set of crystallographic planes containing a common [111] axis be identified by the angle $\phi$, which fixes the inclination of the plane (in the right-handed sense about.[111]) relative to the $[\overline{2} 11]$ direction. - This set of boundaries possesses the point symmetry subgroup $\mathrm{D}_{3 \mathrm{~h}}$. Considering the symmetry reduces the range of physically distinctive inclination angles to $0 \leq \phi \leq \pi / 3$.

Elements of the hypersurface for $<111>$ tilt boundaries consist of a pair of crystallographic planes, sharing a common [111] axis, defined by crystallographic inclinations $\phi_{1}$ and $\phi_{2}$, and joined together such that their crystallographic misorientation is a pure rotation about [111] of angle $\Delta \phi=\left|\phi_{1}-\phi_{2}\right|$. Again, from the energetic point of view it does not matter which of the two crystallographic planes has crystallographic inclination $\phi_{1}$ and which has inclination $\phi_{2}$. The hypersurface $\mathcal{H}_{<111>}$ defining all pure $<111>$ tilt boundaries is therefore given by the set:

$$
\mathcal{H}_{<111>}=\left\{\left(\phi_{1}, \phi_{2}\right): 0 \leq \phi_{1} \leq \pi / 3,0 \leq \phi_{2} \leq \phi_{1}\right\}
$$

Herring's Relations on $\mathcal{H}_{<100>}$ and $\mathcal{H}_{<111 \geq}$ : Relation (1), when applied to $\mathcal{H}_{<100>}$ and $\mathcal{H}_{<111>}$, becomes

$$
\sum_{j=1}^{3}\left\{\sigma\left(\phi_{1}{ }^{j}, \phi_{2}{ }^{j}\right) \hat{b}_{j}+\left(\partial \sigma\left(\phi_{1}{ }^{j}, \phi_{2}{ }^{j}\right) / \partial \bar{\phi}\right)_{\Delta \phi} \hat{n}_{j}\right\}=\overrightarrow{0}
$$


The inclination is $\bar{\phi}=\left(\phi_{1}+\phi_{2}\right) / 2$, and the derivatives are taken at constant misorientation $\Delta \phi$.

\section{A NEW STATISTICAL MULTISCALE METHOD FOR THE EXTRACTION OF THE FREE- ENERGY FUNCTION}

One would expect that if mesostructural data were available for a sufficient number of equilibrated triple junctions, it would be possible to solve relations (4) for the free energy function. (The six unit vectors in (4) obtain directly from experimental measurements of the orientation of the triple line and the three dihedral angles.) Consider, for example, $M$ triple junctions comprising $3 \mathrm{M}$ interfaces. For $\mathcal{H}_{<100>}$ and $\mathcal{H}_{<111>}$ these junctions have parallel line directions. Suppose that the set of $3 \mathrm{M}$ interfaces includes $\mathrm{Q}$ distinctive types. Thus the number of unknown energies and torques in the set is $2 \mathrm{Q}$. Herring's relations provide $\mathrm{K}<2 \mathrm{M}$ independent equations inter-relating these $2 \mathrm{Q}$ unknowns. Clearly if $2 \mathrm{Q} \leq \mathrm{K}$ a solution for all of the unknowns is possible (to within an unknown normalization factor). Note, however, that direct solutions may be both unfeasible and unstable.

Experimental scans will provide data for a vast number of equations of the form (4). Described here is a combination of statistical and multiscale methods which may be exploited to manage this vast amount of data. For purposes of exposition, our attention is restricted to microstructures sufficiently near $\mathcal{H}_{<100>}$ and $\mathcal{H}_{<111>}$ where the energy $\sigma$ is assumed a function of lattice misorientation $\Delta \phi$ alone. Under these circumstances (4) reduces to the force balance consisting of three (Young's) relations

$$
\frac{\sigma_{1}}{\sin \chi_{23}}=\frac{\sigma_{2}}{\sin \chi_{31}}=\frac{\sigma_{3}}{\sin \chi_{12}}
$$

where each equation involves two $\sigma$ 's and hence two values of $\Delta \phi$.

Standard least square analysis is not appropriate to this problem. Discretize the range of $\Delta \phi$ into $N$ subintervals, say, $\left[0, \frac{1}{N} \pi\right),\left[\frac{1}{N} \pi, \frac{2}{N} \pi\right), \ldots,\left[\frac{N-1}{N} \pi, \pi\right]$, and also tabulate the equations. The values $\left(\Delta \phi_{1}, \Delta \phi_{2}\right)$ occurring in a given equation in (4) typically fall into two of these intervals.

Establish $\left(\begin{array}{c}N+1 \\ 2\end{array}\right)=\mathrm{N}(\mathrm{N}+1) / 2$ urns for the pairs $\left(\Delta \phi_{1}, \Delta \phi_{2}\right)$ and place each equation into its respective urn, imposing the statistical hypothesis that each such placement is an independent trial. The Law of Large Numbers then permits us to average the equations in each urn to obtain a set of $\left(\begin{array}{c}N+1 \\ 2\end{array}\right)$ averaged equations. If $\mathrm{N}$ is large, e.g., $\mathrm{N}=256$, there obtains 32,896 equations, still a large number for numerical resolution. Thus it is useful to introduce a multiscale iterative 
method. Begin with a small $N$ and solve the small system for a trial energy, say $\sigma^{\mathrm{N}}$. $\mathrm{N}$ is then increased to $2 \mathrm{~N}$, the data set is resorted and averaged, and resolution at level $2 \mathrm{~N}$ is initiated with the solution $\sigma^{\mathrm{N}}$ of the previous level. By adapting a Kaczmarz method, sparseness of the coefficient matrix is preserved and a solution found rapidly.

Figure 2 shows the result of this iteration for a simulated trial consisting of $1.3 \times 10^{5}$ triple junctions or $3.9 \times 10^{5}$ equations. This simulation, accurate to within $0.5 \%$, required less than 4 minutes on a workstation.

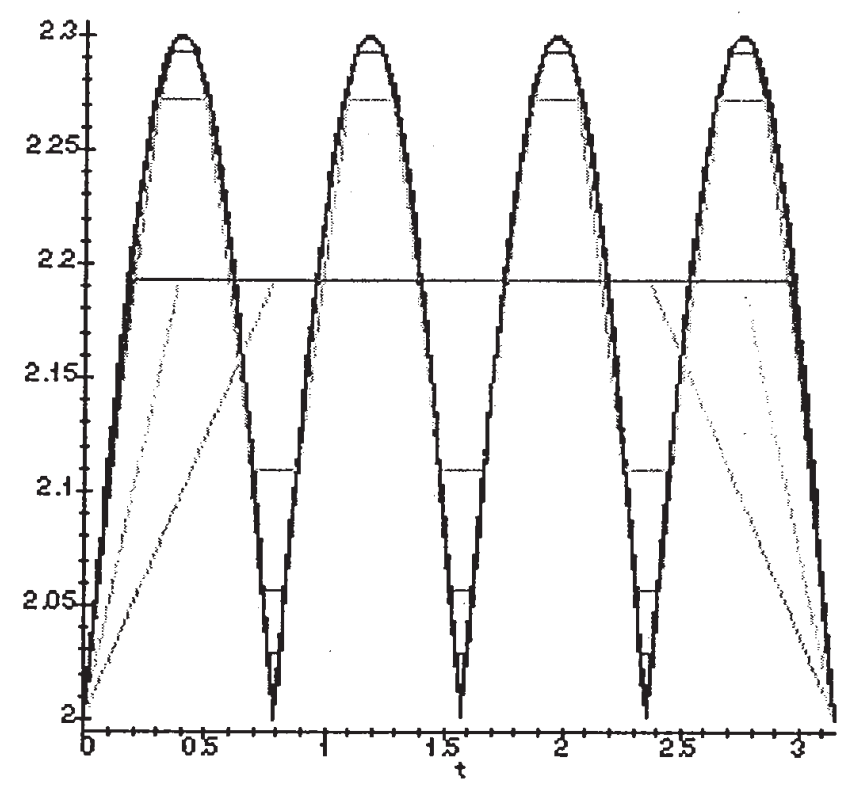

Figure 2. Simulation of the recovery of the free-energy function from the force balance relations. (Shown here is the reconstruction, level by level, for eight scales, beginning with $\mathrm{N}=2$ and ending with $\mathrm{N}=256$.)

\section{SUMMARY REMARKS}

OIM, when coupled with precision serial polishing, is now capable of revealing the 3dimensional structure of polycrystals to resolutions of about $100 \mathrm{~nm}$. When the temperatures and times of equilibration have been sufficient, the mesostructure revealed by this tomography can be expected to satisfy Herring's force and torque balance relations at triple junctions. Thus, the mesostructure carries data required to extract a mapping relating grain boundary excess free energy to the character of the grain boundaries over the full fundamental zone. This approach defines a new inverse problem in materials science. 
Analysis has been explored in this paper for two important two-parameter hypersurfaces of the fundamental zone: the sets of pure $<100>$ and $<111>$ tilt boundaries, labeled $\mathcal{H}_{<100>}$ and $\mathcal{H}_{<111>}$ respectively. For such we have explored a new statistical multiscale analysis that employs the Kaczmarz relaxation. The results of testbed simulations conducted to date demonstrate rapid convergence, and flexible, dynamic partitioning of the fundamental zone.

\section{ACKNOWLEDGMENT}

This work was supported primarily by the MRSEC Program of the National Science Foundation under Award Number DMR-9632556.

\section{REFERENCES}

1. C. Herring, in Structure and Properties of Solid Surfaces, R. Gomer and C. S. Smith, eds., 1952, Chicago: University of Chicago Press.

2. B. L. Adams, S. I. Wright and K. Kunze, Metallurgical Transactions, 24A (1993), 819.

3. P. Lejcek and S. Hofmann, CRC Critical Reviews in Solid State and Materials Science, 20 (1995), 1.

4. J. W. Gibbs, The Scientific Papers of J. Willard Gibbs, Vol. 1: Thermodynamics, 1962, New York: Dover Publications.

5. W. W. Mullins, in Metal Surfaces: Structure, Energetics and Kinetics, W. D. Robertson and N. A. Gjostein, eds., 1963, p. 17, Metals Park: The American Society for Metals. 